\title{
Reflex eyeblinks and visual suppression
}

\author{
KAREN A. MANNING, LORRIN A. RIGGS, and JULIEANE K. KOMENDA \\ Brown University, Providence, Rhode Island
}

\begin{abstract}
Voluntary blinks are known to be accompanied by visual suppression that arises, at least in part, through nonretinal processes. We wanted to test the hypothesis that this suppression must arise as a corollary to the voluntary command to blink. We therefore examined visual sensitivity at the time of a blink that does not involve a voluntary command, namely the corneal reflex elicited by a puff of air. We found that sensitivity was reduced during these reflex blinks; the magnitude and time course of suppression were similar to those of voluntary blinks. We therefore adopt the view that visual suppression stems from signals from the common, efferent, blink pathway.
\end{abstract}

We seldom notice our blinks, even though they occur often and produce large changes in the light reaching the retinal receptors. Eyeblinks may occur 10-15 times per minute (Moses, 1975; Ponder \& Kennedy, 1928). The optical effect of each blink is a blackout that lasts $40-200 \mathrm{msec}$ (Doane, 1980; Gordon, 1951; Lawson, 1948) and eliminates contour stimulation while reducing the light entering the eye by almost two log units (Volkmann, Riggs, \& Moore, 1980). Yet, the subjective effect of a blink is minimal (Moses, 1975; Riggs, Volkmann, \& Moore, 1981).

The above remarks apply mainly to the most common blinks, those that are characterized as spontaneous. Such blinks are thought to be centrally programmed, although by involuntary and largely unconscious means. Two other categories of blink have been described: reflex and voluntary (Blount, 1928; Moses, 1975). Reflex blinks are produced involuntarily, but in response to tactile, auditory, proprioceptive, visual, or electrical stimulation. Voluntary blinks are initiated by the subject in response to an external or internal command to blink.

\section{Reflex vs. Voluntary Blinks}

A typical reflex blink is a rapidly occurring, protective closing of the eyelids over the cornea. This takes place without conscious control; once triggered by an environmental stimulus, such a blink occurs automatically. One kind of reflex blink, the corneal reflex, is elicited by mechanical stimulation of free nerve endings in the cornea. The normal response is a bilateral blink. The time from corneal stimula-

These experiments were conducted in the Walter S. Hunter Laboratory of Psychology at Brown University. The research was supported by Grant EY-03169 from the National Eye Institute to Lorrin A. Riggs. We thank F. C. Volkmann and J. T. McIlwain for helpful advice and R. K. Moore, K. DeLucia, and J. W. Kling for their assistance. The authors' mailing address is: Hunter Laboratory of Psychology, Brown University, Providence, Rhode Island 02912. tion to activation of the lid muscles ranges from 25 to $65 \mathrm{msec}$, with little intrasubject variability (Ongerboer de Visser, Mechelse, \& Megens, 1977; Rushworth, 1962). The neural pathways involved in this reflex are short and direct.

In its most simple form, the corneal reflex is a brainstem phenomenon which does not appear to require cortical involvement (Ongerboer de Visser, 1980; also see Hiraoka \& Shimamura, 1977). After corneal stimulation, afferent nerve impulses travel centrally along the ophthalmic division of the trigeminal nerve (V), enter the brainstem at the level of the pons, and descend along the spinal trigeminal tract to the ipsilateral spinal nucleus of the trigeminal nerve. Impulses ascend from this nucleus to the ipsilateral and contralateral facial nuclei in the pons. Finally, the facial nerve (VII) emerges from the brainstem and innervates the ipsilateral orbicularis oculi muscle of the eye, thus completing the efferent path of the corneal reflex. Activation of the palpebral portion of the orbicularis muscle produces a blink. There is an absence of muscle activity in the levator muscle of the lid when the orbicularis muscle is active during lid-closing (Björk \& Kugelberg, 1953; Gordon, 1951).

All blinks use the same efferent pathway, but a voluntary blink must be initiated consciously by the subject. This is thought to require cortical involvement, possibly including parts of the brain that produce lid closure when stimulated electrically (e.g., Moses, 1975). However, the precise cortical locations and the connections between these areas and brainstem are not known. Thus, using as-yet-unknown pathways, a voluntary command to blink feeds onto the common, efferent pathway that must be activated in order to produce lid closure during eyeblinks.

\section{Suppression}

Two lines of evidence have shown that a voluntary blink is accompanied by a significant loss of visual 
sensitivity. The first is the finding that there is a rise of 0.4 to $0.9 \mathrm{log}$ units in the threshold for detection of a visual stimulus even under experimental conditions in which the lids do not impair the delivery of the test stimulus at the time of a blink (Volkmann, Riggs, Ellicott, \& Moore, 1982; Volkmann et al., 1980). The second is the fact that when blinks are made in the more usual situation of bright surroundings, the perceptual effect is remarkably small (Moses, 1975). In fact, a psychophysical matching procedure has shown that a voluntary eyeblink has a dimming effect that is subjectively equivalent to that produced by a 0.4 to $1.0 \mathrm{log}$ unit weaker decrement in Ganzfeld illumination presented to the open eyes (Riggs et al., 1981).

The blink-related suppression revealed in these studies takes place in the absence of visual contours and image movement. Thus, retinal explanations that involve visual masking, or retinal image movement, or smear do not seem relevant. The experimental results suggest instead that vision is suppressed in some way by neural signals that occur as a corollary to blinks. The present experiments provide further evidence on the nature of such suppression.

\section{Purpose}

The purpose of the present experiments was to find out whether suppression accompanied a reflex blink. If visual suppression of nonretinal origin could be shown to accompany corneal reflex blinks, then we could reject the hypothesis that a conscious command to blink was necessary for suppression to occur. If, on the other hand, suppression was not found with reflex blinks, support would be given to the hypothesis that suppression originated as a corollary to the conscious command to blink.

\section{GENERAL METHOD}

\section{Test Conditions}

An optical system was used to present a test stimulus at a range of times before, during, or after a blink. The light reached the retina from behind in order to bypass the lids and optics of the eye. This system has been described previously by Volkmann et al. (1980). Briefly, one end of a fiber optic bundle was directed to the roof of the mouth in order to deliver light from the optical system. Dark-adapted subjects selected a favorable location for the beam of light, typically medial to the canine tooth on the right side, so that light reached the lower, nasal portion of the right eye through the tissues and sinuses intervening between the roof of the mouth and the globe. The subject then saw, in the upper, temporal visual field, a large and diffuse cloud of light that was typically 1.5-2 log units above threshold (Volkmann et al., 1982). A constant position of the light was assured by the use of a bite board to which the fiber optic bundle was clamped. Securely fitting opaque goggles worn by subjects assured that no light entered the front of the eye. The subjects dark-adapted for at least $\mathbf{3 0}$ min prior to each session.

The stimulus, as in previous studies, was a 30 -msec decrement (AI) in the steady level of retinal illumination (l). The amplitude of the decrement was varied over a range of equal steps of $\log (\Delta \mathrm{I} / \mathrm{I})$.

\section{Airpuff Stimulus}

A gentle puff of air was used to elicit corneal reflex blinks. The triggered release of a spring against an air bladder forced air through a plastic tube. One end of the tube fed into a small nozzle inserted into the goggles worn by the subject. Air was directed toward the temporal margin of the cornea. A pressure-sensitive detector built into a branch of the tubing was used to monitor the magnitude and time of occurrence of each puff. The initial increase in air pressure was complete within 15 msec. The puff of air resulted in a reflex blink of relatively constant amplitude and latency for each subject.

\section{EBG}

The electroblepharogram (EBG) was recorded with a pair of standard skin electrodes above and below the eye and amplified with 3-dB corner frequencies of 0.3 and $30 \mathrm{~Hz}$ (PAR 113). The EBG was used to monitor the subject's blink and was differentiated to determine blink onset and to activate trigger circuits when necessary. Three traces on a storage oscilloscope were used to monitor each airpuff, reflex blink, and stimulus decrement. A trial was excluded if a spontaneous blink occurred before or after the reflex blink, or if the trigger circuit failed to activate the test stimulus at the appropriate time.

\section{Psychophysical Procedure}

Visual sensitivity was measured by a two-alternative, forcedchoice procedure (Green \& Swets, 1966) in a constant-stimulus design. Three experimental conditions were used: (1) a reflex blink condition, (2) a voluntary blink condition, and (3) an open-eye, no-blink condition. The open-eye condition is an important control within each experimental session, since it provides the sensitivity measure against which decreases in sensitivity are compared.

In the reflex blink condition, two airpuffs, 3-4 sec apart, were used to elicit a pair of blinks on each trial. The stimulus decrement was presented randomly during either the first or the second blink, and the subject had to judge which blink had been accompanied by the stimulus. Presentation of the airpuff was always preceded by an auditory warning signal. The subjects were instructed to heed this signal, to keep their eyes open, and to maintain their gaze in the same resting position (as if looking straight ahead).

In the voluntary blink condition, no airpuff was involved, but blinks occurred in pairs as the subject made them in response to an auditory cue. Again, the subject had to judge which blink had been accompanied by the stimulus.

To ensure comparability of timing arrangements and experimental conditions, the same equipment for delivering the stimulus was used in the open-eye condition as in the reflex blink condition. However, the air tube to the goggles was disconnected so that no blink was elicited during no-blink trials. ${ }^{1}$

Each test session consisted of 70-90 trials using blink and openeye (no-blink) conditions. The first few trials within a condition presented large-amplitude, easily visible decrements to the subject to familiarize him with the task. Then the amplitude of the decrement was reduced to near-threshold levels and varied every 5 or 10 trials. Normally, three to five amplitudes were needed to find a threshold for each condition and were used in each test session. Over the course of the experiment, stimuli presented to each subject ranged in amplitude from easily detectable to undetectable decrements of light. Within all sessions, the order of experimental conditions, the order of amplitudes of the decrement within conditions, and the presentation of the stimulus during the first or second blink were varied randomly according to the protocol determined before each session. Overall, at least 30 judgments were obtained in Experiments 1 and 2, and at least 20 judgments were obtained in Experiment 3 at each of the amplitudes needed to generate a psychometric function for each condition. Usually four sessions per subject were necessary to obtain the required number of judgments. 
Psychophysical Functions and Suppression

In all experimental conditions, the subject's proportion of correct judgments increased as a function of the stimulus amplitude $(\Delta \mathrm{I} / \mathrm{I})$. When the data are plotted as in Figure 1, the displacement of the function from one condition relative to another is an indication of the change in a subject's sensitivity.

Threshold values of $(\Delta \mathrm{I} / \mathrm{l})$ were determined using a previously described method (e.g., Volkmann et al., 1982). Proportions of correct judgments were first converted to $Z$ scores, and then a linear function was fitted to each set of $\mathrm{Z}$ scores vs. $\log (\Delta \mathrm{I} / \mathrm{I})$ using a method of least squares. We disregard those portions of the data for which test stimuli were extremely strong, or extremely weak. Linear regression was performed using data points at and above the largest value of $\log (\Delta \mathrm{I} / \mathrm{I})$ at which the subjects' judgments were $50 \%$ correct, up to the smallest value of $\log (\Delta \mathrm{I} / \mathrm{I})$ for $100 \%$ correct judgments. (We used the convention that a $\mathrm{Z}$ score of $100 \%$ was arbitrarily assigned a value of halfway down to the next discrete value. In this way, for example, the $100 \%$ values in Experiments 1 and 2 were converted to $98.3 \%$.) Threshold was defined as that value of stimulus decrement associated with $75 \%$ correct on the function (halfway between chance and 100\%). The $\log$ of the ratio of threshold values in the blink and open-eye conditions is a measure of the relative amount of visual suppression present during blink trials.

\section{EXPERIMENT 1}

The purpose of this experiment was to ascertain whether reflex blinks were accompanied by visual suppression. Thus, it was necessary to measure visual sensitivity for stimuli delivered during reflex blinks for comparison with an open-eye (no-blink) measure of sensitivity. Since these subjects had had no prior experience with eyeblink experiments, their sensitivity was also measured under a voluntary blink condition that is known to produce significant amounts of suppression.

\section{Method}

Triggering. Three experimental conditions were used for each subject in Experiment 1: (1) voluntary blink, (2) reflex blink, and (3) open eye. The stimulus was always delivered at the onset of the blink, as indicated by the EBG display. In the voluntary blink condition, the amplified and differentiated EBG was fed into a Schmitt trigger, which allowed the stimulus to be presented immediately. This method of stimulus presentation was used previously to measure suppression during voluntary blinks (Volkmann et al., 1980). In the reflex blink and open-eye conditions, however, the stimulus was triggered through a delay circuit that was actuated by the device that delivered the airpuff. The latency of each subject's corneal reflex was sufficiently constant for a particular delay to be used to present the stimulus at the onset of the blink. This mode of triggering was used because (1) it eliminated the problem of false triggering from electrical interference by the airpuff device, and (2) it permitted the delivery of the test stimulus with variable amounts of delay as was necessary in Experiment 3.

Procedure. Each session began and ended alternately with one or the other of the blink conditions; the open-eye condition occurred between blink conditions. The right eye was always illuminated. The same right eye received the airpuff during reflex blink trials.

\section{Results}

Figure 1 shows the psychophysical functions obtained in Experiment 1. All three subjects are most sensitive when the decrement is presented during the open-eye condition. Despite individual differences,
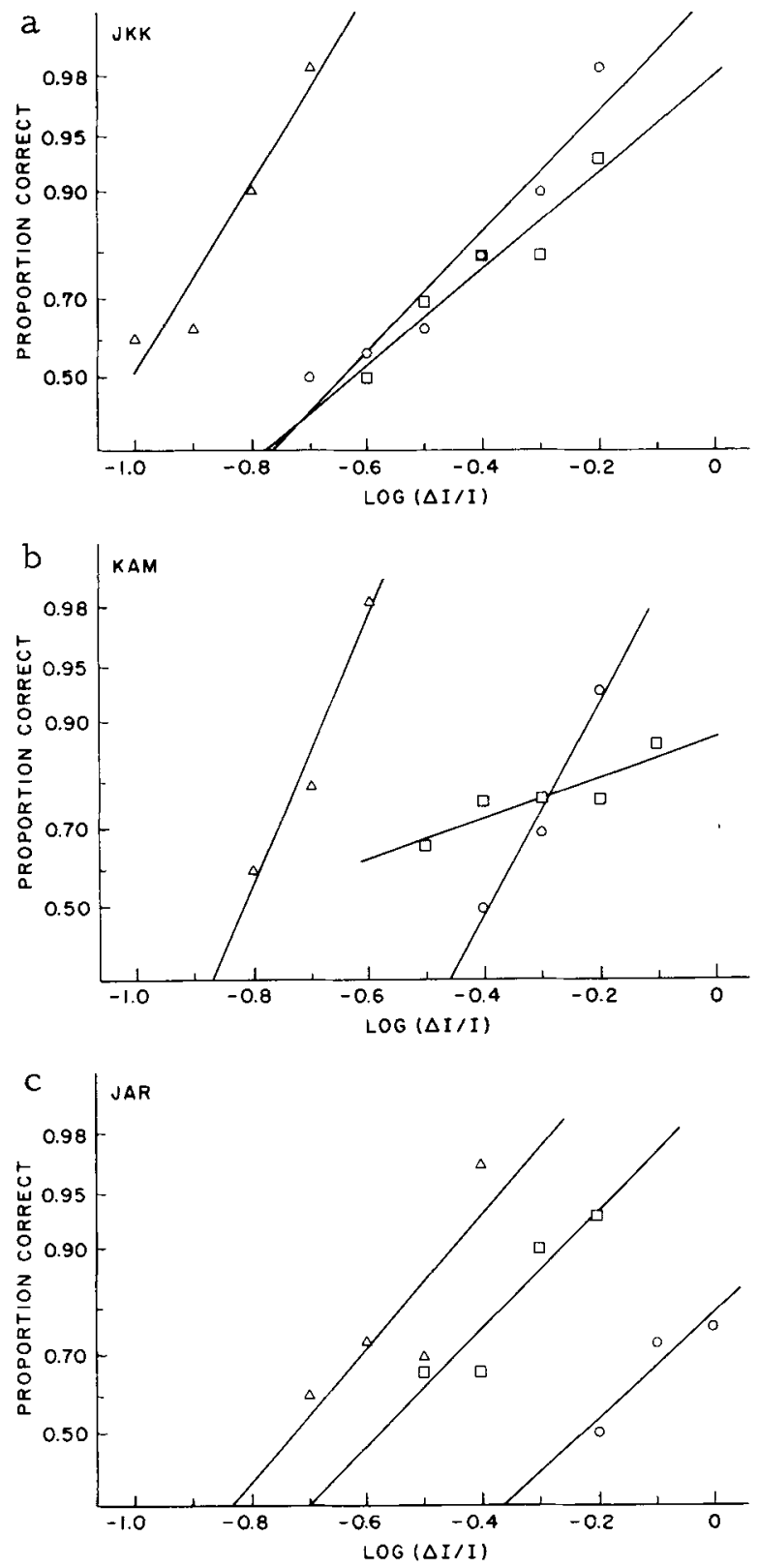

Figure 1. (a) Psychophysical functions from Experiment 1 for Subject J.K.K. The subject's proportion of correct detection of stimulas decrements of variable magnitude in three experimental conditions: open eye (no-blink), $\Delta$; reflex blink, $\square$; and voluntary blink, $O$. In the reflex blink condition, the right eye received both the airpuff stimulus and the decrement of light. The ordinate shows a probability scale and the abscisas shows stimulus magnitude $(\log \Delta I / I)$, where I is the steady retinal illumination and $\Delta I$ is the decrement from this steady level. Linear regression was used to determine a best-fitting line for each function from which a threshold value was derived. For the open-eye, reflex blink, and voluntary blink conditions, respectively, $\mathrm{r}^{2}$ equaled $0.92,0.92$, and 0.92. (b) Psychophysical functions from Experiment 1 for Subject K.A.M. Data plotted as in Figure 1a. The $r^{2}$ value for the open-eye, reflex blink, and voluntary blink conditions equaled $0.96,0.79$, and 0.97 , respectively. (c) Psychophysical functions from Experiment 1 for Subject J.A.R. Data plotted as in Figure 1a. The $r^{2}$ values for the open-eye, reflex blink, and voluntary blink conditions equaled $0.73,0.87$, and 0.86 , respectively. 
it is clear that, for successful detection, all subjects required larger decrements in blink trials than in open-eye trials. In other words, visual sensitivity was impaired at the time of voluntary blinks and reflex blinks.

Table 1 shows the magnitude of visual suppression computed from data in Figure 1. All subjects show roughly 0.5 -log-unit suppression during voluntary blinks; in the reflex blink condition, J.K.K. and K.A.M. show 0.5 and 0.4-log-unit suppression, respectively. Although J.A.R. shows less suppression than the other subjects in the reflex blink condition ( $0.2 \log$ units), it is nevertheless clear that visual suppression is present; there is no overlap of the functions for his open-eye and blink conditions.

\section{EXPERIMENT 2}

The purpose of Experiment 2 was to find out whether suppression would still be found in the reflex blink condition if the airpuff was delivered to the left eye rather than to the same eye (right) in which vision was tested. In this experiment, as in Experiment 1, (1) a reflex blink and (2) an open-eye condition were used. Experimental procedures were exactly the same as those used in the first experiment, except that the airpuff now was directed toward the opposite eye. The amplitude of the EBG was usually similar, regardless of which eye received the airpuff.

\section{Results}

The results of Experiment 2 are shown in Table 1. When the data were analyzed as in Experiment 1, the amounts of suppression measured were nearly identical to those obtained in Experiment 1 for the same three subjects. When the stimulus was presented at blink onset, Subjects J.K.K. and K.A.M. showed 0.5-log-unit suppression and J.A.R. showed 0.2-logunit suppression.

\section{EXPERIMENT 3}

The purpose of Experiment 3 was to measure visual sensitivity for stimuli presented at various times before, during, and after a reflex eyeblink.

Table 1

Reflex and Voluntary Blink Suppression (Log Units)

\begin{tabular}{lllll}
\hline & & \multicolumn{3}{c}{ Subject } \\
\cline { 3 - 5 } Condition & & J.K.K. & K.A.M. & J.A.R. \\
\hline & Experiment & & & \\
Voluntary Blink & 0.42 & 0.44 & 0.54 \\
Reflex Blink (RE puff; RE illum.) & 0.48 & 0.38 & 0.17 \\
\multicolumn{4}{c}{ Experiment 2} \\
Reflex Blink (LE puff: RE illum.) & $\mathbf{0 . 4 9}$ & 0.49 & 0.18 \\
\hline
\end{tabular}

\section{Method}

Triggering. Two experimental conditions were used within each session: (1) reflex blink and (2) open eye. The same method for triggering was used in both conditions. On each trial, the experimenter initiated simultaneously a sequence of events leading to presentation of the visual stimulus and the airpuff stimulus. Both systems had time delays built in that could be independently adjusted. Thus, the airpuff, and therefore the regularly occurring reflex blink, could be set to occur at specific times before, during, or after the decrement.

Procedure. The experimental procedure was similar to that of Experiment 1. Again, the reflex blink was elicited by an airpuff to the right eye, and the same eye was tested for visual sensitivity. Throughout this experiment, however, the test decrement was presented at eight times: at blink onset, at $242,142,75$, and $30 \mathrm{msec}$ prior to blink onset, and at 30,75 , and $142 \mathrm{msec}$ after blink onset. A total of 7 to 11 sessions were needed to complete the experiment. During odd-numbered test sessions, the subjects were tested with 10 trials at each of two different amplitudes of stimulus decrement presented 142 and 30 msec before blink onset and 142 and $30 \mathrm{msec}$ after blink onset. During even-numbered sessions, the stimuli were presented $75 \mathrm{msec}$ before and after blink onset and at blink onset. Each time the delay was changed, the subjects were given a few practice trials at relatively high stimulus values to familiarize them with the specific timing. Throughout the experiment, all times were monitored on the display of the storage oscilloscope.

\section{Results}

The results of Experiment 3 are plotted in Figure 2. Here, sensitivity is plotted as a function of the time of stimulus presentation in relation to the time of reflex blink onset for all three subjects. Sensitivity in the open-eye condition is also shown.

The pattern of results is similar in all cases. Although individual sensitivities differ, all subjects are least sensitive when the stimulus is presented $30 \mathrm{msec}$ prior to blink onset. Sensitivity then increases (i.e., suppression decreases) systematically as the stimulus occurs at longer intervals before or after blink onset. Maximum visual suppression associated with reflex blinks occurred at the same time for J.K.K., K.A.M., and J.A.R., and equaled $0.49,0.71$, and $0.37 \log$ units, respectively.

\section{CONTROL EXPERIMENT}

A control experiment was performed to rule out the possibility that some of the loss of visual sensitivity might have resulted from auditory or tactile stimulation accompanying the airpuff and not from the blink.

\section{Method}

In this experiment, visual sensitivity was measured in the usual way. In one control condition, however, the lower right cheek of Subject K.A.M. received the airpuff so that no blink was elicited. In the other control condition, the airpuff tube was disconnected, as before, so that no puff reached the subject. Within both conditions, two different delay settings were used, one appropriate for delivering the decrement at onset of a reflex blink and the other appropriate for delivering the decrement $30 \mathrm{msec}$ prior to blink onset. 


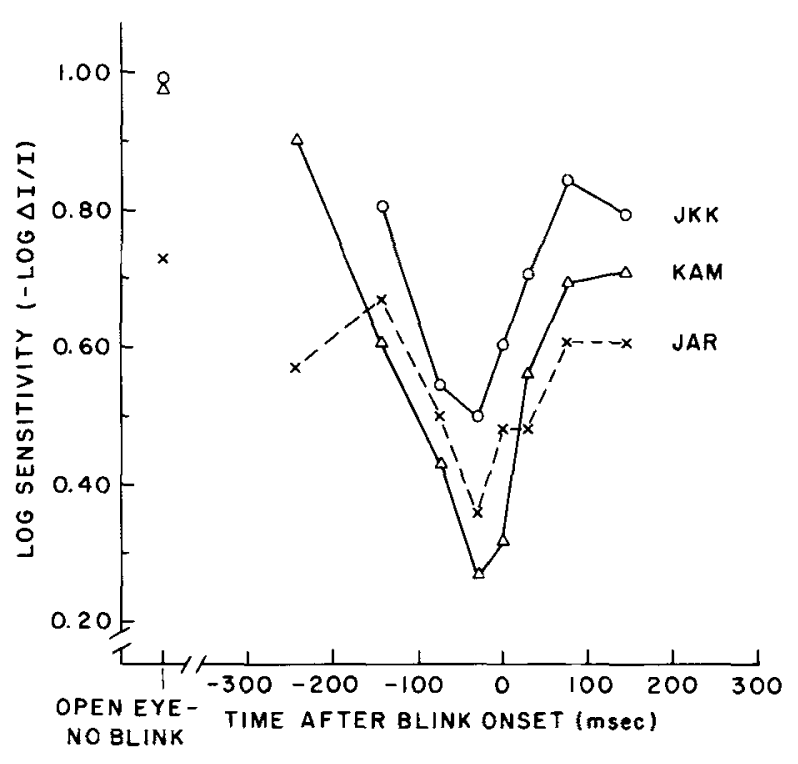

Figure 2. Visual sensitivity before, during, and after onset of reflex blink in Experiment 3. Log sensitivity (the reciprocal of threshold) is plotted as a function of the time the stimulus oceurred after onset of the reflex blink for three subjects. Sensitivity in the open-eye condition is also shown. Each plotted point was derived from a poychophysical function like those in Fignre 1.

\section{Results}

The results of the control procedure are shown in Figure 3, plotted with the fitted lines from Figure $1 \mathrm{~b}$. They provide no evidence that suppression was associated with the use of the airpuff stimulus itself. The four functions from the control procedure overlap each other and also the psychophysical data obtained from the same subject in the open-eye condition of Experiment 1. In none of the cheek-puff control conditions are the functions shifted in sensitivity with respect to no-puff trials as are the functions for reflex and voluntary blink conditions. We therefore conclude that, in the main experiments, the observed changes in visual sensitivity were indeed determined by the presence or absence of a blink.

\section{DISCUSSION}

\section{Outcome of These Experiments}

It is clear that visual suppression accompanies reflex eyeblinks. The subjects were less sensitive by $0.4-$ $0.7 \log$ units to stimuli presented just before the onset of the blink. Even Subject J.A.R., who showed only 0.2-log-unit suppression when the stimuli were presented at onset of the reflex blink, showed 0.4-logunit suppression to stimuli presented $30 \mathrm{msec}$ before blink onset. ${ }^{2}$ For all subjects, nearly identical amounts of suppression were measured, regardless of whether the same eye or the opposite eye received the initial puff of air.
The control procedure addressed the possibility that visual suppression during reflex blinks might somehow result from competing tactile or auditory effects of the airpuff stimulus. But no appreciable decrease in sensitivity was found when the puff was applied to the cheek (still stimulating the trigeminal nerve) instead of the cornea. Thus, the $0.7-\log$-unit loss of sensitivity that accompanies this subject's reflex blink cannot be attributed to extraneous stimuli or to any simple loss of attention.

The amounts of suppression measured in these reflex blink experiments are roughly the same as those occurring with voluntary blinks. In addition, the time course of the suppression associated with a reflex blink resembles closely the one associated with a voluntary blink (Volkmann et al., 1980).

\section{Source of Suppression}

The conditions under which the present experiments were conducted (light entering the eye diffusely from behind the retina) provide no possibility that retinal image movement, smear, or masking can account for blink-related elevation of visual threshold. Earlier reports of experiments using similar conditions (Riggs et al., 1981; Volkmann et al., 1982; Volkmann et al., 1980) have summarized the evidence that a feed-forward type of neural signal is produced with the efferent activity that initiates lid lowering and acts to reduce visual sensitivity. Evidence has been adduced (Volkmann et al., 1982) that

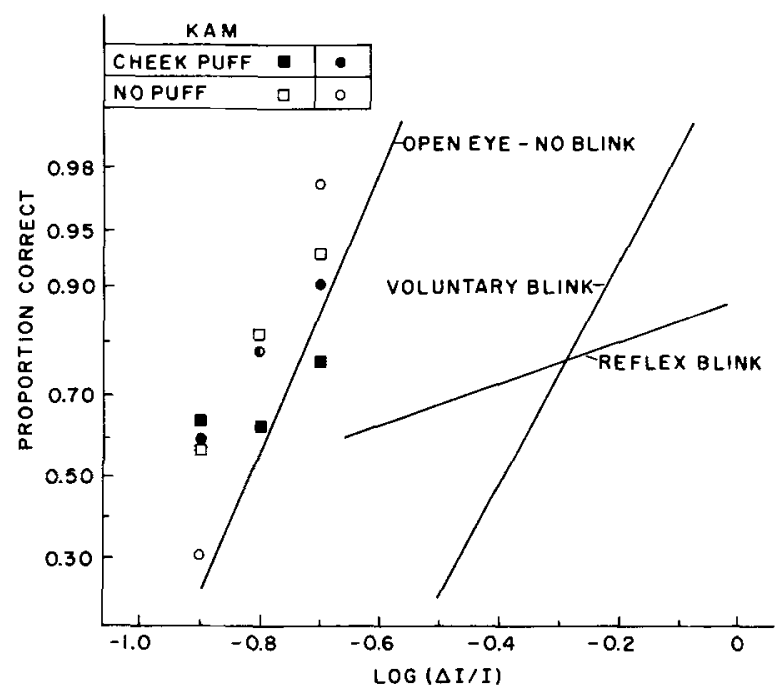

Figure 3. Psychophysical datn from the cheek-puff control procedure. For reference, the fitted lines from Figure 1b are shown. In the control procedure, two conditions were used: cheek-puff (filled symbols) and no-puff (open symbols). Two different airpuff delay times were included within both basic conditions: 0 msec (circles) and 30 msec (squares). The data from the control experiment in which no blinks occurred are consistent with those obtained in the open-eye condition (fitted line) from the same subject (K.A.M.) in Experiment 1. 
this cannot be ascribed to the suppression that is known to accompany saccadic eye movements. Such small eye movements as take place during a blink do not appear to be saccades, but more passive, nasalward motions-attributable to forces from the closure of the lids (Doane, 1980; Ginsborg \& Maurice, 1959; Hung, Hsu, \& Stark, 1977; Miller, 1967; Volkmann et al., 1980). The present results support the concept of an efferent corollary signal, but also indicate that its suppressive effect upon visual sensitivity need not arise from a conscious command to blink.

There are striking examples of efferent signals in other species that modify sensory processing. These signals act to reduce an animal's sensitivity to the sensory consequences of its motor activities. In fish and frogs, stimulation of the lateral line, which would occur from ripples in the water generated by the animal's movement, is suppressed by an efferent discharge that occurs as a corollary to movement. Efferent fibers impinge directly on afferent neurons and reduce sensitivity by an amount that may reach 1-2 log units, depending on the vigor of the motion (Russell, 1976). A similar control mechanism is present in the auditory system of bats. A discharge of the vocalization muscles causes ear muscles to contract and also produces neural attenuation in the central, auditory pathway (Suga \& Schlegel, 1972). In one species of electric fish, an efferent signal that is a corollary to the motor command to fire the electric organ appears to reduce the central, neural effects evoked by electric organ discharge (Bell, 1981). In the locust, an efferent corollary discharge that is concomitant with saccadic eye movements reduces the response of a visual neuron in the brain by nearly $1 \mathrm{log}$ unit (Zaretsky, 1982). The off-response of one class of retinal ganglion cells in the toad fails to occur when the animal closes its eyelids, and an inhibitory, efferent signal that accompanies the command to close the lids is believed to be responsible (Borchers \& Ewert, 1978). Finally, there is preliminary evidence of inhibition of cat visual cortical neurons at the time of a blink (Buisseret \& Maffei, 1983). Since this occurs under conditions in which the lid does not obscure the pupil, it suggests that inhibition is produced through central, neural processes.

The animal experiments demonstrate widespread examples of discharges that could heighten the organism's responsiveness to environmental stimuli by minimizing the sensory effects of its own actions. If efferent activity of this kind is present in human subjects, it could serve to maintain perceptual stability by reducing the otherwise disruptive blackouts of the visual scene during blinks.

\section{REFERENCES}

Beus, C. C. An efference copy which is modified by reafferent input. Science, 1981, 214, 450-453.
Blount, W. P. Studies of movements of the eyelid of animals: Blinking. Quarterly Journal of Experimental Physiology, 1928, 18, 111-125.

Björk, A., \& Kugelberg, M. D. The electrical activity of the muscles of the eye and eyelids in various positions and during movement. Electroencephalography and Clinical Neurophysiology, 1953, 5, 595-602.

Borchers, H. W., \& Ewent, J. P. Eye closure in toads (Bufo bufo $L$.) does not produce off responses in retinal on-off ganglion cells: A question of efferent commands. Journal of Comparative Physiology, 1978, 125, 301-308.

Buisseret, P., \& MAfFet, L. Suppression of visual cortical activity during eyeblinks in the cat. Journal of Physiology (London), 1983, 334, 19P. (Abstract)

DoAnE, M. G. Interaction of eyelids and tears in corneal wetting and the dynamics of the normal human eyeblink. American Journal of Ophthalmology, 1980, 89, 507-516.

Ginsbora, B. L., \& Maurice, D. M. Involuntary movements of the eye during fixation and blinking. British Journal of Ophthalmology, 1959, 43, 435-437.

Gordon, G. Observations upon the movements of the eyelids. British Journal of Ophthalmology, 1951, 35, 339-351.

Green, D. M., \& Swets, J. A. Signal detection theory and psychophysics. New York: Wiley, 1966.

Hiraoka, M., \& Shimamura, M. Neural mechanisms of the corneal blinking reflex in cats. Brain Research, 1977, 125, 265-275.

Hung, G., Hsu, F., \& Stark, L. Dynamics of the human eyeblink. American Journal of Optometry and Physiological Optics, 1977, 54, 678-690.

LAwson, R. W. Blinking: Its role in physical measurement. Nature, 1948, 161, 154-157.

Miller, D. Pressure of the lid on the eye. Archives of Ophthalmology, 1967, 78, 328-330.

Moses, R. A. The eyelids. In R. A. Moses (Ed.), Adler's physiology of the eye (6th ed.). St. Louis: Mosby, 1975.

Ongerboer de Visger, B. W. The corneal reflex: Electrophysiological and anatomical data in man. Progress in Neurobiology, 1980, 15, 71-83.

Ongerboer de Vigser, B. W., Mechelse, K., \& Megens, P. H. A. Corneal reflex latency in trigeminal nerve lesions. Neurology (Minneapolis), 1977, 27, 1164-1167.

Ponder, E., \& Kennedy, W. P. On the act of blinking. Quarterly Journal of Experimental Physiology, 1928, 18, 89-110.

Riggs, L. A., Volkmann, F. C., \& Moore, R. K. Suppression of the blackout due to blinks. Vision Research, 1981, 21, 10751079.

Rushworth, G. Observations on blink reflexes. Journal of Neurology, Neurosurgery and Psychiatry, 1962, 25, 93-108.

Russell, I. J. Amphibian lateral line receptors. In R. Llinas \& W. Precht (Eds.), Frog neurobiology-A handbook. Berlin: Springer, 1976.

Suga, N., \& Schlegal, P. Neural attenuation of responses to emitted sounds in echo locating bats. Science, 1972, 177, 82-84.

Volkmann, F. C., Rrggs, L. A., Ellicott, A. G., \& Moore, R. K. Measurements of visual suppression during opening, closing, and blinking of the eyes. Vision Research, 1982, 22, 991 996.

Volkmann, F. C., Rtgos, L. A., \& Moone, R. K. Eyeblinks and visual suppression. Science, 1980, 207, 900-902.

ZARETgKY, M. Quantitative measurements of centrally and retinally generated saccadic suppression in a locust movement detector neuron. Journal of Physiology (London), 1982, 328, 521-533.

\section{NOTES}

1. Since the subject could perceive the same warning signal and incidental sounds in the open-eye condition as during the 
reflex blink condition, the question arises whether a conditioned reflex blink might be developed. However, no lid response was observed with the EBG monitoring device, which was carefully watched.

2. Throughout these experiments, J.A.R. consistently showed less reflex blink suppression than did the other subjects. The amplitude of J.A.R.'s reflex blinks, measured with high-speed motion picture records, was small in comparison with that of his voluntary blinks. The records of Subjects J.K.K. and K.A.M. showed nearly the same amplitude of voluntary and reflex blinks. It seems likely that the amount of suppression may be correlated with blink amplitude.

(Manuscript received March 3, 1983;

revision accepted for publication May 31, 1983.) 